\title{
Estudo de Características Quantitativas de Crescimento dos 120 aos 550 Dias de Idade em Gado Nelore
}

\section{João Cláudio do Carmo Paneto1, Daniela Cristina Lemos², Luiz Antônio Framartino Bezerra ${ }^{3}$, Raimundo Martins Filho ${ }^{4}$, Raysildo Barbosa Lôbo ${ }^{5}$}

\begin{abstract}
RESUMO - Visando melhor entendimento das características quantitativas ligadas ao crescimento do gado Nelore, foi realizado estudo das estimativas de componentes de variância e coeficientes de herdabilidade para ganhos de peso nas idades padronizadas de 120 a 240,240 a 365,365 a 455 e 455 a 550 dias e ganhos de perímetro escrotal nas idades padronizadas de 365 a 455 e 455 a 550 dias. Componentes de covariância e correlações entre as características de ganho de peso e ganho de perímetro escrotal também foram estimados. Foram utilizados 29.769 registros de ganhos de peso e 15.676 registros de ganhos de perímetro escrotal de animais participantes do PMGRN, entre os anos de 1986 e 1999. As estimativas dos componentes de (co)variância foram obtidas pelo método da máxima verossimilhança restrita, com algoritmo livre de derivadas, sob modelo animal. As estimativas obtidas foram: 0,32 de herdabilidade direta e 0,13 de herdabilidade materna para ganho de peso entre 120 e 240 dias de idade; 0,16, 0,21 e 0,23 de herdabilidade direta para ganho de peso nas idades de 240 a 365, 365 a 455 e 455 a 550 dias, respectivamente; 0,24 e 0,18 de herdabilidade direta para ganho de perímetro escrotal nas idades de 365 a 455 e 455 a 550 dias, respectivamente; e correlação genética de 0,18 entre os ganhos de peso e de perímetro escrotal.
\end{abstract}

Palavras-chave: bovinos, componentes de (co)variância, ganhos de perímetro escrotal, ganhos de peso, Nelore

\section{Study of Quantitative Growth Traits from 120 to 550 Days of Age in Nellore Cattle}

ABSTRACT - With the aim of a better understanding of quantitative growth traits in Nellore cattle, some parameters were estimated: variance components and heritability coefficients of weight gains between the standardized ages of 120 and 240,240 and 365,365 and 455 , and 455 and 555 days, and of scrotal circumference gains between the standardized ages of 365 and 455, and 455 and 555 days; and (co)variance components and genetic correlations between weight and scrotal circumference gains. Information from 29,769 records of weight gain and 15,676 records of scrotal circumference gain were analyzed to obtain the (co)variance component estimates. The restricted maximum likelihood, derivative free, under an animal model was the method of choice. The results were the following: 0.32 for the direct genetic heritability and 0.13 for the maternal genetic heritability coefficients for weight gain between 120 and 240 days of age; $0.16,0.22$ and 0.23 for the direct genetic heritability coefficients for weight gain between the ages of 240 and 365,365 and 455 , and 455 and 550 days respectively; 0.24 and 0.18 for the direct genetic heritability coefficients for scrotal circumference gain between the ages of 365 and 455 , and 455 and 550 respectively; and genetic correlation of 0.18 between the weight and the scrotal circumference gains.

Key Words: cattle, (co)variance components, Nellore, scrotal circumference gain, weight gain

\section{Introdução}

A produção de carne de forma eficiente é o principal objetivo das criações domésticas de bovinos de corte. Assim, o estudo detalhado das fases de ganho de peso mostra como as condições genéticas e de manejo podem influenciar a produção desses animais. O perímetro escrotal é um indicador de algumas características genéticas de interesse econômico, incluindo ganho de peso e fertilidade de machos e fêmeas. Ambas as características, ganho de peso e ganho de perímetro escrotal, estão relacionadas aos objetivos de seleção na maioria dos programas de melhoramento de bovinos de corte, sendo importantes critérios a serem avaliados para permitir escolhas mais adequadas.

Para o criador, quanto mais rápida for a taxa de crescimento dos animais, mais curto será o ciclo de seus produtos, podendo reduzir os custos de manutenção na propriedade, principalmente se o sistema de produção for o confinamento. Isso significa que o rendimento deve ser baseado em quantidade de ani-

\footnotetext{
1 Doutorando, FMRP-USP / Professor, UNIUBE. Av. Nenê Sabino, 1801, sala I-04, CEP 38055-500, Uberaba-MG. E.mail: paneto@yahoo.com

2 Bolsista CNPq/aluna doutorado FMRP-USP.E.mail: dclemos@genbov.fmrp.usp.br

3 Analista de sistemas do Departamento de Genética, FMRP-USP. E.mail: lafbezer@genbov.fmrp.usp.br

4 Professor Adjunto da UFC - Fortaleza - CE. E.mail: martins@ufc.br

5 Professor Associado, Departamento de Genética, FMRP-USP. E.mail: rayblobo@genbov.fmrp.usp.br
} 
mais vendidos jovens e não em animais vendidos mais velhos e mais pesados (Marcondes, 1999).

As pressões exercidas pelo mercado e a necessidade de se produzir carne bovina de boa qualidade, de forma eficiente e competitiva, têm estimulado pecuaristas e pesquisadores a buscarem alternativas de critérios de seleção para precocidade, tanto de crescimento como sexual. No entanto, esses conceitos não podem ser utilizados indistintamente, pois certamente essas características estão muito correlacionadas (Garnero, 1999).

O cálculo dos ganhos de peso nas fases pré- e pós-desmame auxilia o processo seletivo dos animais, pois demonstra o potencial de velocidade em ganho de peso, e também possibilita a escolha de animais mais precoces (Garnero, 1999; Marcondes, 1999). O ganho de peso pré-desmame é fortemente influenciado pela habilidade materna da vaca, enquanto o ganho de peso pós-desmame representa o potencial individual de crescimento, porém, com influência ambiental considerável (Marcondes, 1999). O ganho de peso na fase imediatamente após o desmame pode sofrer grande influência ambiental. Dependendo da época do nascimento, esse período coincide com o início da estação seca, o que traz escassez de alimentos para animais criados a pasto e redução na média de ganho de peso, em decorrência dessa escassez.

O desempenho reprodutivo dos rebanhos é um dos principais fatores determinantes da eficiência total de produção da bovinocultura de corte, devendo, de acordo com Alencar et al. (1993), ser considerado nos programas de melhoramento genético. Esses mesmos autores sugerem a fertilidade dos machos como critério de seleção alternativo para aumentar a taxa de natalidade imediata e permanente, uma vez que a baixa herdabilidade das características de fertilidade das fêmeas aliada à baixa intensidade de seleção, normalmente aplicada a esse sexo, resulta em baixo progresso genético esperado.

O perímetro escrotal é um parâmetro fácil de ser medido e passou a ser característica valiosa na seleção quando Coulter \& Foote (1979) e Bourdon \& Brinks (1986), em reprodutores Bos taurus taurus, observaram correlações positivas com a produção de sêmen, fertilidade e características de produção, como peso. Bergmann et al. (1996) também encontraram correlações genéticas positivas entre pesos e perímetros escrotais. De acordo com Lôbo (1996), o perímetro escrotal possibilita a seleção por ser uma característica de fácil obtenção e a magnitude do coeficiente de herdabilidade ser relativamente alta (0,30 a 0,40). Sanches \& Lôbo (1998) observaram que os animais da raça Nelore, que atingiram a puberdade mais cedo, possuíam, em média, perímetros escrotais superiores entre os de seu grupo. Hoje em dia, o emprego do perímetro escrotal para compor os critérios de seleção em bovinos de corte tem sido muito utilizado na maioria dos programas de melhoramento em todo o mundo (Garnero, 1999).

Os objetivos deste trabalho foram estimar os componentes de (co)variância para efeitos genéticos diretos dos ganhos de peso entre as idades padronizadas de 120 e 240, 240 e 365, 365 e 455, 455 e 550 dias, e dos ganhos de perímetro escrotal entre as idades padronizadas de 365 e 455 e 455 e 550 dias; estimar os componentes de (co)variância para efeitos genéticos maternos para o ganho de peso pré-desmame, dos 120 aos 240 dias de idade; e estimar as correlações genéticas entre ganho de peso e ganho de perímetro escrotal. Com essas informações, pretende-se avaliar a possibilidade de utilização dessas características como critérios de seleção em rebanhos bovinos da raça Nelore.

\section{Material e Métodos}

O arquivo de dados analisado consistia em 29.769 registros de ganhos de peso e 15.676 registros de ganhos de perímetro escrotal em várias fases do crescimento, indo desde os 120 até os 550 dias de idade para peso e dos 365 aos 550 dias de idade para perímetro escrotal, sendo todos os animais pertencentes ao Programa de Melhoramento Genético da Raça Nelore (PMGRN) USP. Os animais estudados nasceram entre os anos de 1986 e 1999. As pesagens foram feitas a cada 90 dias (janeiro, abril, julho e outubro) e ao desmame de cada lote. As características estudadas foram $\mathrm{GP}_{120-240}$ (ganho de peso total entre as idades padronizadas de 120 e 240 dias), $\mathrm{GP}_{240-365}$ (ganho de peso total entre as idades padronizadas de 240 e 365 dias), GP $_{365-455}$ (ganho de peso total entre as idades padronizadas de 365 e 455 dias), GP $_{455-550}$ (ganho de peso total entre as idades padronizadas de 455 e 550 dias), GPE $_{365-455}$ (ganho de perímetro escrotal total entre as idades padronizadas de 365 e 455 dias) e $\mathrm{GPE}_{455-550}$ (ganho de perímetro escrotal total entre as idades padronizadas de 455 e 550 dias). Constam na Tabela 1 os números de observações, médias, mínimos, máximos, desvios-padrão e coeficientes de variação das características formadoras do arquivo de dados analisado. 
Tabela 1 - Número de observações (N), médias aritméticas, mínimos (MIN), máximos (MAX), desvios-padrão (DP) e coeficientes de variação $(\mathrm{CV})$ das características estudadas

Table 1 - Number of records (N), means, minimum (MIN), maximum (MAX), standard deviations (SD) and coefficients of variation (CV) for the studied traits

\begin{tabular}{lcccccc}
\hline Característica & $\mathrm{N}$ & Média & MIN & MAX & DP & CV $(\%)$ \\
Trait & $N$ & Mean & MIN & MAX & SD & \\
\hline $\mathrm{GP}_{120-240}{ }_{1}^{1}(\mathrm{~kg})$ & 26039 & 66,94 & $-3,00$ & 173,00 & 19,76 & 29,52 \\
$\mathrm{GP}_{240-365}(\mathrm{~kg})$ & 22126 & 35,61 & $-42,00$ & 167,00 & 23,64 & 66,37 \\
$\mathrm{GP}_{365-455_{1}}(\mathrm{~kg})$ & 18161 & 40,09 & $-30,00$ & 142,00 & 18,32 & 45,71 \\
$\mathrm{GP}_{455-550}(\mathrm{~kg})$ & 15086 & 44,26 & $-48,00$ & 148,00 & 19,32 & 43,65 \\
$\mathrm{GPE}_{365-455^{2}}(\mathrm{~mm})$ & 4230 & 34,60 & $-36,00$ & 109,00 & 14,56 & 42,09 \\
$\mathrm{GPE}_{455-550}(\mathrm{~mm})$ & 4629 & 32,61 & $-38,00$ & 127,00 & 12,99 & 39,83 \\
\hline
\end{tabular}

1 GP - ganho de peso total entre as idades padronizadas indicadas (Total weight gain between the indicated standardized ages).

${ }^{2} \mathrm{GPE}$ - ganho de perímetro escrotal total entre as idades padronizadas indicadas (Total scrotal circumference gain between the indicated standardised ages).

Os rebanhos participantes do PMGRN localizam-se nos Estados de Goiás, Mato Grosso do Sul, Minas Gerais, São Paulo, Maranhão e Bahia. A maioria das fazendas, ao ingressar no PMGRN, adota estação de acasalamento, cuja duração varia de 60 a 90 dias dentro do período de outubro a março, conforme a região e o nível de manejo do rebanho. A maioria dos animais é alimentada exclusivamente a pasto. É freqüente a utilização da inseminação artificial, com repasse de touros, após a primeira ou segunda oportunidade. O desmame dos bezerros é feito entre os sete e oito meses de idade (Garnero, 1999).

As análises genéticas foram feitas no laboratório de computação do bloco $\mathrm{C}$ do Departamento de Genética da Faculdade de Medicina de Ribeirão Preto/USP, por intermédio do programa MTDFREML (Boldman et al., 1995), sob sistema operacional DOS. Assim, as estimativas dos componentes de (co)variância foram obtidas pelo método de máxima verossimilhança restrita, com algoritmo livre de derivadas, sob um modelo animal.

Os parâmetros genéticos estimados para as características $\mathrm{GP}_{120-240}, \mathrm{GP}_{240-365}, \mathrm{GP}_{365-455}$, $\mathrm{GP}_{455-550}, \mathrm{GPE}_{365-455}, \mathrm{GPE}_{455-550}$ foram:

1. variâncias genéticas aditivas diretas $\left(\sigma_{\mathrm{d}}^{2}\right)$, maternas $\left(\sigma_{\mathrm{m}}{ }^{2}\right.$; somente para $\left.\mathrm{GP}_{120-240}\right)$, residuais $\left(\sigma_{\mathrm{e}}{ }^{2}\right)$ e fenotípicas $\left(\sigma_{\mathrm{p}}^{2}\right)$;

2. coeficientes de herdabilidade direta $\left(\mathrm{h}_{\mathrm{d}}{ }^{2}\right)$ para todas as características estudadas, e materna $\left(\mathrm{h}_{\mathrm{m}}{ }^{2}\right)$ somente para ganho de peso total entre as idades padronizadas de 120 e 240 dias (GP $120-240)$;

3. covariâncias e correlações genéticas entre os ganhos de peso e ganhos de perímetros escrotais.
Em termos de notação matricial, o modelo estatístico para $\mathrm{GP}_{120-240}$, que inclui efeito materno, foi:

$$
\mathrm{y}=\mathrm{Xb}+\mathrm{Z}_{1} \mathrm{a}+\mathrm{Z}_{2} \mathrm{~m}+\mathrm{e}
$$

$\mathrm{O}$ modelo para as características de ganho de peso pós-desmame e ganho de perímetro escrotal, que não inclui efeito materno, foi:

$$
\mathrm{y}=\mathrm{Xb}+\mathrm{Z}_{1} \mathrm{a}+\mathrm{e}
$$

em que: y - vetor das observações; X - matriz de incidência dos efeitos fixos; $b$ - vetor dos efeitos fixos; $Z_{1}$ - matriz de incidência dos efeitos aleatórios genéticos diretos; a - vetor dos efeitos aleatórios genéticos diretos; $Z_{2}$ - matriz de incidência dos efeitos aleatórios genéticos maternos; $\mathrm{m}$ - vetor dos efeitos aleatórios genéticos maternos; e - vetor dos erros aleatórios.

Os efeitos fixos utilizados foram grupos de contemporâneos e classe de idade da vaca ao parto. Os grupos de contemporâneos foram compostos pelas seguintes informações: fazenda, ano de nascimento, sexo do animal, trimestre de nascimento e grupo de manejo concatenado (contemplando os grupos em que o animal esteve após os 120 dias de idade). As idades da vaca ao parto foram agrupadas em seis classes: menor que 36, de 36 a 47, de 48 a 59, de 60 a 71 , de 72 a 119 e maior que 119 meses de idade.

A consistência dos dados e a avaliação estatística dos modelos e efeitos fixos utilizados foram realizadas com o uso do pacote estatístico SAS para Windows, versão 6.12 (SAS, 1996). Foram eliminados animais com diferenças de mais de três desvios padrão da média de seu grupo e considerados apenas grupos de contemporâneos com mais de três animais. 


\section{Resultados e Discussão}

Para visualização e melhor entendimento das fases de crescimento para as quais os parâmetros genéticos foram estimados neste trabalho, o gráfico da Figura 1 apresenta, a partir dos 120 dias de idade, as médias de ganhos de peso diário durante os quatro períodos de crescimento estudados.

No período de 120 a 240 dias, observa-se média de ganho de $0,56 \mathrm{~kg} /$ dia. A habilidade de ganho de peso nessa fase depende bastante da habilidade materna por ser um período pré-desmame. No período imediatamente posterior ao desmame, 240 a 365 dias de idade, percebe-se queda na velocidade de crescimento, quando foi observada média de ganho de $0,28 \mathrm{~kg} / \mathrm{dia}$. Os nascimentos ocorrem entre os meses de julho e dezembro e, conseqüentemente, os desmames ocorrem entre os meses de fevereiro e agosto. Sendo assim, a maior parte dos desmames ocorre no início da estação seca, e os animais tendem a ganhar menos peso, ou mesmo perder peso nesse período. A habilidade genética para ganhar peso nessa fase deve estar relacionada à habilidade de ganho de peso em condições adversas, mostrando maior adaptabilidade dos animais que apresentam bons resultados nessa fase. No período seguinte, 365 a 455 dias de idade, percebe-se nova ascensão na velocidade de crescimento, $0,45 \mathrm{~kg} / \mathrm{dia}$, refletindo o início do período de estação das águas e a disponibilidade de forrageiras. Geneticamente, deve demonstrar nos animais a habilidade direta de desenvolvimento em condições favoráveis e/ou de ganho compensatório. O último período estudado, 455 a 550 dias de idade, demonstra a habilidade genética individual

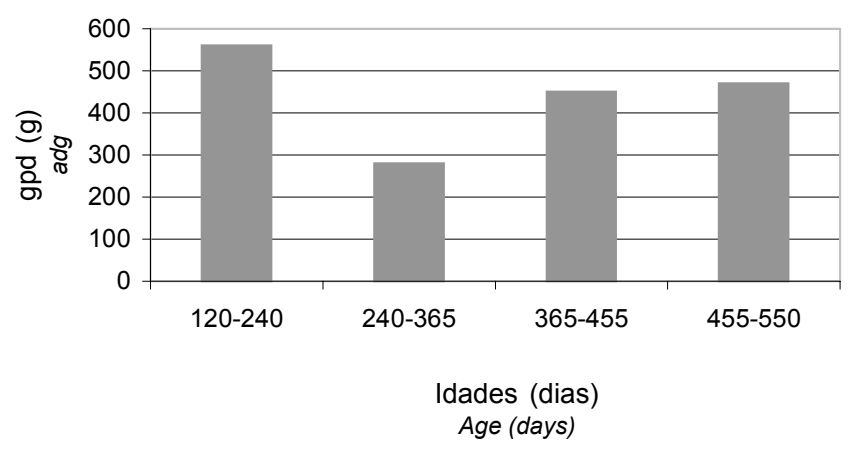

Figura 1 - Ganho de peso diário (gpd) em quatro períodos de crescimento.

Figure 1 - Average daily gain (adg) in four growth periods. de desenvolvimento ponderal em condições favoráveis. Nessa fase, manteve-se velocidade de crescimento próxima àquela do período anterior, $0,47 \mathrm{~kg} /$ dia. A observação dessa figura mostra que a velocidade de ganho de peso é variável com o período de crescimento. Isso indica que padronizações que ajustam o peso para idades determinadas, feitas por regressão linear, podem estar embutindo erros nas análises realizadas. Estudos mais específicos devem indicar qual o tipo de ajuste mais adequado para cada circunstância.

Os componentes de variância estimados para as características em análises unicaráter estão apresentados, para as características pré- e pós-desmame, na Tabela 2.

Garnero (1999), revisando 31 estudos na literatura, encontrou a média de 0,25 para o coeficiente de herdabilidade direta de características de crescimento pré-desmame. Para herdabilidade materna, Garnero et al. (1998) encontraram valores de 0,00 e 0,15 para ganho de peso diário em diferentes fases do crescimento pré-desmame, sendo que o enfoque foi a medição do ganho de peso diário até determinado peso, diferentemente do presente estudo. A estrutura de coleta dos dados, dentro do PMGRN, é bastante eficiente para essa fase e a pesagem ao desmame é feita na maioria dos animais. Dessa maneira, justificam-se os valores de herdabilidade encontrados acima da média da literatura, 0,32 para herdabilidade direta e 0,13 para herdabilidade materna. A correlação entre os efeitos genéticos direto e materno foi $-0,71$. Valores negativos, para esse parâmetro, foram encontrados em outros estudos para pesos e ganhos de peso e taxa de crescimento pré-desmame (Meyer, 1994; Garnero et al., 1998; Ortiz Peña, 1998; Reyes et al., 1997; Marcondes, 2000). O valor de herdabilidade direta encontrado neste estudo para $\operatorname{GP}_{120-240}(0,32)$ indica a possibilidade de utilização dessa característica como critério de seleção. A herdabilidade materna para $\operatorname{GP}_{120-240}(0,13)$ mostra a influência do efeito genético materno nessa fase. A correlação negativa encontrada entre esses dois efeitos reforça a importância de inclusão do efeito materno nas análises genéticas. Marcondes (1999) comenta a necessidade de inclusão dos efeitos maternos nos programas de seleção, dizendo ainda que isso traria resultados mais confiáveis, a longo prazo, do que a seleção baseada unicamente no efeito genético direto.

Para ganho de peso do desmame a um ano de 
Tabela 2 - Estimativas dos componentes de variância e herdabilidade Table 2 - Variance components and heritability estimates

\begin{tabular}{|c|c|c|c|c|c|c|}
\hline $\begin{array}{l}\text { Característica } \\
\text { Trait } \\
\end{array}$ & $\sigma_{d}^{2}$ & $\sigma_{\mathrm{m}}^{2}$ & $\sigma_{\mathrm{e}}^{2}$ & $\sigma_{p}^{2}$ & $\mathrm{~h}_{\mathrm{d}}^{2}$ & $\mathrm{~h}^{2}{ }_{\mathrm{m}}$ \\
\hline $\mathrm{GP}_{120-240}{ }^{1}$ & 57,96 & 22,90 & 125,39 & 180,28 & 0,32 & 0,13 \\
\hline $\mathrm{GP}_{240-365}$ & 30,06 & - & 161,99 & 192,05 & 0,16 & - \\
\hline $\mathrm{GP}_{365-455}$ & 34,08 & - & 123,74 & 157,82 & 0,21 & - \\
\hline $\mathrm{GP}_{455-550}$ & 41,58 & - & 142,41 & 184,00 & 0,23 & - \\
\hline $\mathrm{GPE}_{365-455^{2}}$ & 35,20 & - & 110,55 & 145,75 & 0,24 & - \\
\hline $\mathrm{GPE}_{455-550}^{2}$ & 23,23 & - & 105,13 & 128,86 & 0,18 & - \\
\hline
\end{tabular}

1 GP - ganho de peso total entre as idades padronizadas indicadas (Total weight gain between the indicated standardized ages).

2 GPE - ganho de perímetro escrotal total entre as idades padronizadas indicadas (Total scrotal circumference gain between the indicated standardised ages). $\sigma_{d}^{2}, \sigma_{m}^{2}, \sigma_{e}^{2}, \sigma_{p}^{2}, h_{d}^{2}$ e ${ }^{2}{ }_{m}$ - componentes de variância genética aditiva direta e aditiva materna, residual e fenotípica total, e herdabilidades direta e materna, respectivamente (Direct additive, maternal aditive, residual and phenotypic variance components, direct and maternal heritability coefficients, respectively).

idade, Biffani et al. (1998) encontraram o valor de $0,24 \pm 0,06$ para o coeficiente de herdabilidade direta. Em oito estudos revisados, Garnero (1999) encontrou o valor médio de 0,28 para a herdabilidade direta. Marcondes (1999) encontrou um coeficiente de herdabilidade direta de 0,18 para essa mesma característica. Essa é uma fase do crescimento em que os animais deste estudo sofreram bastante influência do meio sobre seu desenvolvimento, pois a maioria foi desmamada no início da estação seca e sofreu restrição alimentar nesse período. Tem-se, ainda, em virtude da mudança na velocidade de crescimento, a possibilidade de maiores erros na padronização que foi feita por ajuste linear. Em virtude da menor média de ganhos de peso nesse período, observou-se coeficiente de variação muito alto $(66 \%)$ quando foi feita análise descritiva dessa característica. Provavelmente por causa desses fatores, a característica $\mathrm{GP}_{240-365}$ apresentou coeficiente de herdabilidade 0,16 , que está abaixo da maioria dos trabalhos encontrados na literatura. Esse coeficiente indica que essa característica apresentaria resposta pequena se utilizada como critério de seleção nesse rebanho.

Os coeficientes de herdabilidade para ganho de peso em idades posteriores a 365 dias são, na média dos valores da literatura, em torno de 0,21 (Garnero, 1999). Os valores encontrados nesse estudo não diferem da literatura e foram de 0,21 e 0,23 para $\mathrm{GP}_{365-455} \mathrm{e} \mathrm{GP}_{455 \text { - }}$ 550 , respectivamente. Esses coeficientes são de magnitude média, indicando que, se essas características forem utilizadas como critérios de seleção, podem trazer aumento no ganho de peso do período correspondente.

Para ganho de perímetro escrotal, os valores de herdabilidade direta encontrados, 0,24 e 0,18 para os dois períodos estudados, $\mathrm{GPE}_{365-455}$ e $\mathrm{GPE}_{455-550 \text {, }}$ respectivamente, sugerem a possibilidade de ganhos genéticos a partir da seleção baseada nessas características. Alencar et al. (1993), estudando o crescimento do perímetro escrotal dos 12 aos 18 meses de idade, encontraram o valor de 0,21 para herdabilidade direta e também sugeriram a possibilidade de seleção para essa característica.

No entanto, como apontado por Alencar et al. (1993), os perímetros escrotais e os ganhos de perímetro escrotal em idades imediatamente superiores, como perímetro escrotal aos 12 meses de idade e ganho de perímetro escrotal dos 12 aos 18 meses, por exemplo, podem apresentar correlações genéticas e fenotípicas negativas, indicando que animais precoces sexualmente podem apresentar crescimento testicular reduzido após certa idade, por já terem atingido bom tamanho testicular. Bergmann et al. (1996), estudando perímetro escrotal aos 12 e aos 18 meses de idade, em gado Nelore, encontraram maior variabilidade genética para o perímetro escrotal aos 18 meses. Nesse estudo, eles apresentam uma representação gráfica do crescimento testicular e ficam caracterizados um período de crescimento linear, próximo aos 12 meses, e um período de crescimento com tendência curvilínea, próximo aos 18 meses, indicando a proximidade da maturidade sexual dos animais. Os animais que atingem a proximidade da maturidade sexual mais cedo tendem a apresentar bom crescimento testicular até os 12 meses de idade e crescimento testicular reduzido após esse período. Assim, a seleção de animais pelo crescimento testicular dos 12 aos 18 meses de idade poderia causar diminuição do perímetro escrotal aos 12 
meses e, por conseqüência, diminuição da precocidade sexual do rebanho.

Os coeficientes de herdabilidade obtidos nas análises bicaráter não apresentaram diferenças quando comparados com os obtidos nas análises unicaráter. $\mathrm{Na}$ Tabela 3 são apresentados os resultados das correlações genéticas e fenotípicas entre ganhos de peso e ganhos de perímetro escrotal entre as idades padronizadas de 365 e 455 e 455 e 550 dias.

Neste estudo, as análises bicaráter, envolvendo os ganhos de peso e de perímetro escrotal, em dois períodos do desenvolvimento dos animais, demonstraram correlação genética baixa, porém positiva, entre essas características, 0,18 para ambos os casos (Tabela 3). Dessa maneira, os resultados sugerem a ausência de conflito quando se faz seleção simultânea para ganho de peso e ganho de perímetro escrotal.

Valores altos (acima de 0,61) de correlação genética entre pesos e perímetros escrotais após o desmame foram encontrados por Alencar et al.(1993), indicando que a seleção para peso resultaria em aumento no perímetro escrotal. Lôbo et al. (1995) encontraram correlações positivas entre perímetro escrotal aos 365 e 550 dias de idade e peso aos 240 dias de idade, de 0,57 e 0,42 , respectivamente. Os resultados obtidos por Quirino \& Bergmann (1998) para correlação entre perímetro escrotal e peso corporal também foram positivos e variaram de 0,33 a 0,71 , sugerindo que a seleção para perímetro escrotal deve resultar em aumento do peso corporal.

Os resultados não permitem inferir qual seria a resposta sobre o peso dos animais se fosse feita seleção para ganho de perímetro escrotal. Sabe-se apenas que as correlações entre peso e perímetro escrotal e entre ganho de peso e ganho de perímetro escrotal são positivas. Para se chegar a alguma conclusão a esse respeito, seria necessário outro estudo para verificação da correlação entre ganho de perímetro escrotal e peso.

Tabela 3 - Estimativas de correlações, genética $\left(r_{g}\right)$ e fenotípica $\left(r_{p}\right)$ entre ganhos de peso e ganhos de perímetro escrotal, de acordo com a idade

Table 3 - Estimates of genetic $\left(r_{g}\right)$ and phenotypic $\left(r_{g}\right)$ correlations between weight gains and scrotal circumference gains, according to the age

\begin{tabular}{lcc}
\hline $\begin{array}{l}\text { Idade } \\
\text { Age }\end{array}$ & $\mathrm{r}_{\mathrm{g}}$ & $\mathrm{r}_{\mathrm{p}}$ \\
\hline 365 a 455 & 0,18 & 0,17 \\
455 a 550 & 0,18 & 0,24 \\
\hline
\end{tabular}

\section{Conclusões}

O ganho de peso pré-desmame, medido pelo $\mathrm{GP}_{120-240}$, apresentou coeficiente de herdabilidade que sugere a possibilidade de sua utilização como critério de seleção, devendo-se, no entanto, atentar para a correlação negativa existente entre os efeitos genéticos direto e materno.

O ganho de peso do desmame aos 365 dias, $\mathrm{GP}_{240-365}$, sofreu muita influência do ambiente, não sendo um bom critério de seleção.

A correlação genética entre os ganhos de peso e de perímetro escrotal foi de magnitude baixa, porém positiva, indicando a possibilidade de resposta correlacionada favorável quando se seleciona em qualquer das duas características, ou ainda, a possibilidade de seleção simultânea nas duas características.

\section{Agradecimento}

FINEP/BID, FAPESP, CNPq/Rhae, PRONEX, ANCP e criadores do PMGRN.

\section{Literatura Citada}

ALENCAR, M.M.; BARBOSA, P.F.; BARBOSA, R.T.et al. Parâmetros genéticos para peso e circunferência escrotal em touros da raça Canchim. Revista da Sociedade Brasileira de Zootecnia, v.22, n.4, 572-583, 1993.

BERGMANN, J.A.G.; ZAMBORLINI, L.C.; PROCÓPIO, C.S.O. Estimativas de parâmetros genéticos do perímetro escrotal e do peso corporal em animais da raça Nelore. Arquivo Brasileiro de Medicina Veterinária e Zootecnia, v.48, n.1, p.69-78, 1996.

BIFFANI, S.; MARTINS FILHO, R.; BOZZI, R. et al. Parâmetros genéticos e fenotípicos para características de crescimento em animais da raça Nelore. In: REUNIÃO ANUAL DA SOCIEDADE BRASILEIRA DE ZOOTECNIA, 35., 1998, Botucatu. Anais... Botucatu: Sociedade Brasileira de Zootecnia, 1998. p.428-430.

BOLDMAN, K.G.; KRIESE, L.A.; Van VLECK, L.D. et al. A manual for use of MTDFREML: a set of programs to obtain estimates of variance and covariance [DRAFT]. Lincoln: Agricultural Research Service, 1995.120p.

BOURDON, R.M.; BRINKS, J.S. Scrotal circumference in yearling Hereford bulls: adjustment factors, heritabilities and genetic, environmental and phenotypic relationships with growth traits. Journal of Animal Science, v.62, n.4, p.958-967, 1986.

COULTER, G.H.; FOOTE, R.H. Testicular mensuration in bulls as indication of reproductive performance and their relation with the productive characteristics: a review. Theriogenology, v.11, p.297-311, 1979.

GARNERO, A.V.; LÔBO, R.B.; REYES, A. et al. Estimativas de parâmetros genéticos para características incluídas em critérios de seleção em gado de corte. In: REUNIÃO ANUAL DA SOCIEDADE BRASILEIRA DE ZOOTECNIA, 35., 
1998, Botucatu. Anais... Botucatu: Sociedade Brasileira de Zootecnia, 1998. p.434-436.

GARNERO, A.V. Comparação de critérios de seleção em gado corte visando precocidade de crescimento. Ribeirão Preto: Universidade de São Paulo, 1999. 85p. Dissertação (Mestrado em Genética) - Universidade de São Paulo, 1999.

LÔBO, R.B. Programa de melhoramento genético da raça Nelore. 3.ed. Ribeirão Preto: Universidade de São Paulo, 1996. 88p.

LÔBO, R.B.; REYES, A. de los.; BEZERRA, L.A.F. et al. Parâmetros fenotípicos e genéticos de pesos e perímetro escrotal às idades-padrão em animais da raça nelore. In: REUNIÃO ANUAL DA SOCIEDADE BRASILEIRA DE ZOOTECNIA, 32., 1995, Brasília. Anais... Brasília: Sociedade Brasileira de Zootecnia, 1995. p.625-627.

MARCONDES, C.R. Análise de alguns critérios de seleção para características de crescimento na raça Nelore. Belo Horizonte: Universidade Federal de Minas Gerais, 1999. 93p. Dissertação (Mestrado em Zootecnia) - Universidade Federal de Minas Gerais, 1999.

MARCONDES, C.R. Análise de alguns critérios de seleção para características de crescimento na raça Nelore. Arquivo Brasileiro de Medicina Veterinária e Zootecnia, v.52, n.1, p.83-89, 2000.

MEYER, K. Estimates of direct and maternal correlations among growth traits in Australian beef cattle. Livestock Production Science, v.38, p.91-105, 1994.

ORTIZ PEÑA, C.D. Análise de critérios de seleção para precocidade sexual e de crescimento de bovinos da raça Nelore, no Paraguai. Jaboticabal: Universidade Estadual Paulista, 1998. 104p. Dissertação (Mestrado em Zootecnia) Universidade Estadual Paulista, 1998.
QUIRINO, C.R.; BERGMANN, J.A.G. Heritability of scrotal circumference adjusted and unadjusted for body weight in Nellore bulls, using univariated and bivariated animal models. Theriogenology, v.49, p.1389-1396. 1998.

REYES, A.B.; LÔBO, R.B.; BEZERRA, L.A.F. et al. Variabilidade genética de características do crescimento alternativas para a seleção em gado de corte. In: REUNIÃO ANUAL DA SOCIEDADE BRASILEIRA DE ZOOTECNIA, 34., 1997, Juiz de Fora. Anais... Juiz de Fora: Sociedade Brasileira de Zootecnia, 1997. p.245-247.

SANCHES, A.C.; LÔBO, R.B. Precocidade em touros zebuínos jovens. Avaliação genética de animais jovens, touros e matrizes. Ribeirão Preto: Universidade de São Paulo, 1998. $56-57$.

SAS INSTITUTE. Statistical Analysis System. 6.12 versão para Windows ${ }^{\circledR}$. Cary: 1996.

Recebido em: 04/12/00

Aceito em: 07/11/01 